\title{
Structural Colors for Display and E-paper Applications
}

\author{
L. Jay Guo \\ Department of Electrical Engineering and Computer Science \\ The University of Michigan, Ann Arbor, Michigan, USA
}

\begin{abstract}
Light interacting with micro- and nano- structures can produce various optical phenomena. Such properties can be exploited to produce structural colors without using colorant pigments. Potential applications in energy-saving displays and e-papers can be envisioned by integrating with LCD or electrophoretic modulation schemes.
\end{abstract}

\section{Author Keywords}

Structural colors; color pigments; spectrum filters; plasmonics; LCD color filters; light recycling; image sensor; colored epapers.

\section{Introduction}

Color filters have been widely used as key elements in applications ranging from light emitting diodes/image sensors to liquid crystal display and colored e-book/paper technologies. Currently colorant pigmentation are widely employed in various display technologies. The chemical pigments are vulnerable to a variety of processing chemicals, cannot withstand constant illumination with strong light intensities, and require extensive multilayer processing to pattern individual pixels. In nature, colors can also be produced by light interacting with physical structures [1,2], e.g. the blue color of the Morpho butterflies is due to the photonic crystal-like volume-diffractive nanostructures on its wings [3]. Inspired by these natural phenomena, structural colors can be produced by exploiting light interaction with photonic crystals [4] and plasmonic nanostructures $[1,5,6]$ that gives rise to selective transmission or reflection of particular light wavelength. These new structures hold the promise of compatibility with fabrication technology, compact structures and multi-functional usages. Recent developments in large area nanofabrication have opened efficient way to control light properties at subwavelength scale. This paper reviews some of the progress in this field.

\section{Compact and high efficiency plasmonic spectrum filters with polarization control}

Surface plasmon-based nanostructures are attractive due to their small dimensions and the ability of efficient light manipulation. We have shown that by selective conversion between the freespace waves and spatially confined modes in plasmonic nanoresonators formed by the subwavelength metal-insulator-metal (MIM) stack arrays, the transmission spectra through such arrays can be well controlled by using simple design rules. An ultrathin plasmonic nano-grating is capable of filtering white light into individual colors across the entire visible band. The color filters made by these plasmonic resonators are capable of transmitting arbitrary colors with transmission of $\sim 60 \%$ [7]. The key concept here is to use the nano-grating to realize the photon-plasmon-photon conversion efficiently at specific resonance wavelengths. Compared with other spectral filtering methods [5,6], this new design significantly improves absolute transmission, polarization and pass bandwidth.
The device is designed as a subwavelength periodic metalinsulator-metal (MIM) stack array on a magnesium fluoride $\left(\mathrm{MgF}_{2}\right)$ transparent film. Specifically this structure utilize the coupled surface plasmon waves at the two metal-dielectric interfaces. For each stack, a $100 \mathrm{~nm}$-thick zinc selenide $(\mathrm{ZnSe})$ layer is sandwiched by two $40 \mathrm{~nm}$-thick aluminium (Al) layers. The $100 \mathrm{~nm}$-thick ZnSe layer ensures the efficient coupling of SP modes at the top and bottom edges of the stack, whereas the 40 nm-thick Al layer prohibits the direct transmission of the incident light. The bottom Al grating is used to couple selectively the incident light into plasmon waveguide modes by diffraction, whereas the top $\mathrm{Al}$ grating efficiently reconverts the confined plasmons to propagating waves by scattering and transmits the light to the far field in the forward direction. By combining different MIM stack arrays, arbitrary nano-micrometer-scale colorful patterns can be obtained. At present, this lateral dimension is 1-2 orders of magnitude smaller than the best highdefinition color filters currently available. Furthermore, these plasmonic devices have longitudinal thickness $1 \sim 2$ orders of magnitude thinner than the colorant ones, which is very attractive to the design of ultrathin panel display devices.

Besides the small dimensions, the nature of polarization dependence of plasmonic resonators also greatly benefits the applications in LCD display by eliminating the need of a separate polarizer layer.

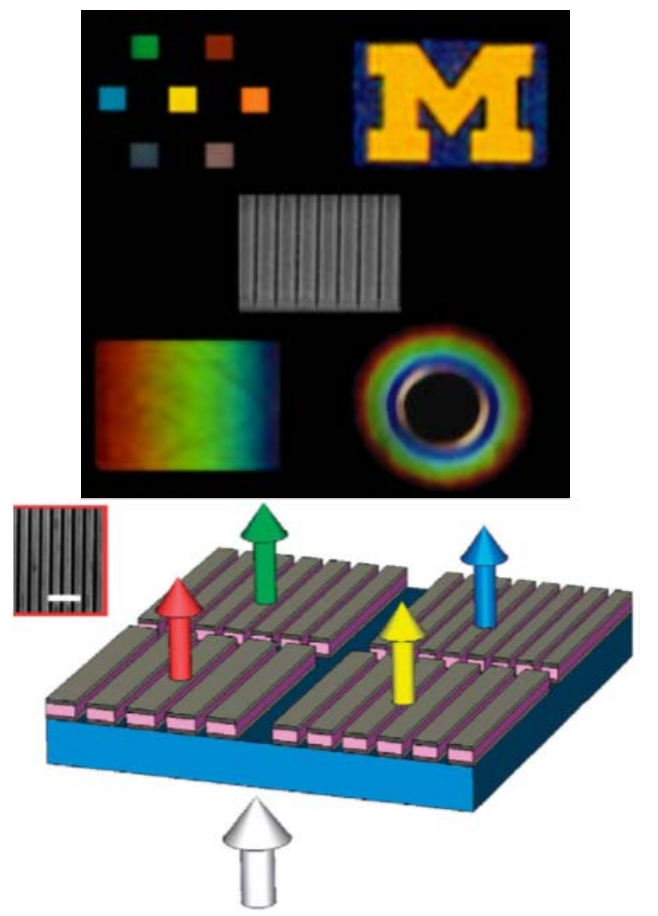

Figure 1. Plasmonic color filter made of nanogratings of MIM stack, capable of generating any color in the visible band by simply choosing different period; and experimental demonstration of colored pixels, an $\mathrm{M}$ logo of 20 micron size and disperse of spectra by varied period. 


\section{High transmittance and tunable color purity filters using single-layer metallic nanograting}

High peak transmission and efficiency of a thin-film color filter is of primary interest. Depending on the specific applications some technologies desire simultaneous polarization of the transmitted light while others may require narrow spectral peaks for high purity colors. For large-scale production, one can employ nanoimprint lithography to fabricate these structures over large areas. For this purpose we have designed alternative structure in the form of metal resonance waveguide grating (MRWG) that are better suited for such fabrication process. Importantly, the design of the MRWG structure also allows for control over transmission bandwidth and the ability to reduce individual filters down to micron-scale pixel sizes. Apart from various display applications, these properties can be particularly useful in compact spectrum analysis devices, as well as image sensor technology where high peak transmission is a necessity while scaling and bandwidth control can allow for variations in pixel size and color filter array architectures [7].

The device structure, as shown in Fig. 2(a), consists of two dielectric layers deposited on a glass substrate with a wide linewidth metal grating on top. Representative transmission spectra for RGB filters are shown in Fig. 2(b). Including the glass substrate, the three dielectrics form a waveguide for light of specific frequency band. The "waveguide" layer is a high index material such as $\mathrm{Si}_{3} \mathrm{~N}_{4}$ while the "buffer" layer is a low index material $\mathrm{SiO}_{2}$ matched to the glass substrate. Since the propagation constant of the guided modes can be provided by the first order scattering of incident light by the metal grating, we can choose the proper grating period to efficiently couple the incident light into waveguide modes at a specific resonant wavelength. A nother distinct advantage of the proposed MRWG filter is that it can be easily tuned from a narrow to a wide bandwidth filter by controlling the "buffer" layer thickness. Details can be found in Ref. 8. The ability to tailor the spectral bandwidth is particularly useful in display and image sensor applications. Currently LCDs use LEDs as their backlight units. The ability of bandwidth control allows our color filters to be designed to match the LED spectrum, which maximizes the
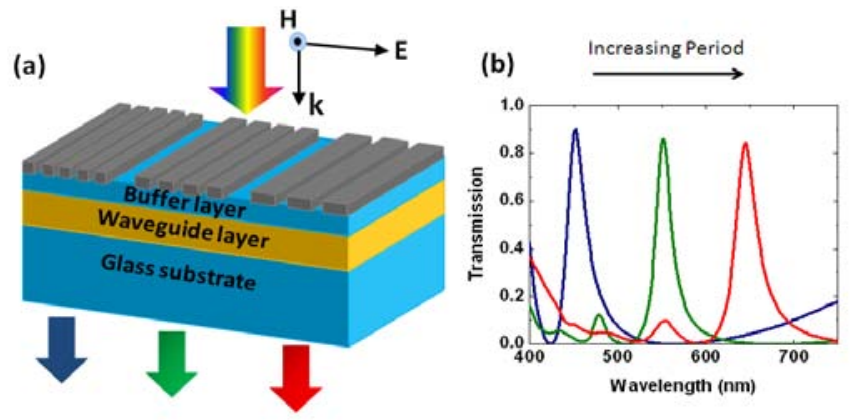

(c)

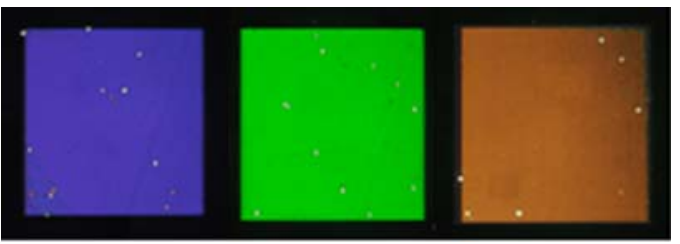

Figure 2. (a) Schematic of the transmissive MRWG color filter structure and (b) Simulated transmission spectra and (c) microscope images of the structures illuminated by white light. overall light utilization and meanwhile reduces possible crosstalks.

Before proceeding further, we would like to summarize several attractive characteristics of these types of color filter:

1) Color filtering is by optical resonance rather than optical absorption; the non-transmitted light can be reflected and potentially recycled to improve LCD efficiency.

2) The color filter is polarization dependent: it transmits TM polarized light, but reflecting TE light; making it suitable to integrate with liquid crystal control to turn the pixel on and off. The reflected TE light can be reflected and recycled.

3) The subwavelength metal grating itself can act as transparent electrode for controling the rotation of LCf in LCD displays [9].

4) Similar design principle can be expanded to reflective color filter, allowing the display device to operate under direct sun light.

5) The structures allow bandwidth control (especially with the MRWG filter discussed above), and therefore offer flexible design for different backlight sources to match the emission band and increase the overall efficiency.

6) The MIM stacks utilize two metal layers sandwiching a dielectric layer. Such a structure is fully compatible with photovoltaic structure if the two metal layers can be used simultaneously as electrode and the dielectric layer replaced by semiconductors. The structure can transmit specific color with high efficiency. The absorbed light will produce photocurrent rather being wasted to heat. This concept was illustrated in Ref. 10

\section{Angle-independent reflection color filters based on vertical nanocavity arrays}

For metallic nanostructures discussed above, the transmitted colors show angle dependence, which is directly related to resonance excitation via grating coupling. This approach has led to high coupling efficiencies, but is inherently angle-dependent due to momentum matching conditions. Such iridescence of the above devices need to be addressed for practical applications of structural colors in certain display applications.

In contrast to grating coupling, plasmonic based resonators and antennas have been demonstrated as candidates for structure colors $[11,12]$. In order to increase the scattering efficiency and generate more vivid colors, we employ a vertical plasmonic resonator array with specially designed periodicity to avoid angle variation. In this design, light funneling into nanoslits is exploited to generate strong absorption for transverse magnetic (TM) polarized incident light. Utilizing this approach, extremely small physical dimensions have large scattering cross sections, and therefore possess plasmonic mode coupling with efficiency comparable to that of grating coupling. We theoretically and experimentally investigated angle-robust structures with near-perfect absorption, as large as $96 \%$, in the visible spectrum. Moreover, wide color tunability throughout the entire visible spectrum and pixel size beyond the diffraction limit were demonstrated [13]. Light of specific wavelength that satisfies the resonance condition can be trapped inside the nanoslits and energy dissipated, while the rest of the light are reflected from the metal surface, giving rise to distinct colors. The most straightforward design of obtaining different colors is by changing the depth of the slits. But from the fabrication point of view, it will be more desirable to keep the same depth 
but varying the slit width. Figure 3 shows the SEM picture of the metallic nanoslits arrays and the colored images of the fabricated structures in the form of Olympic Rings based on the above principle, as well as the angle-dependence for different period of the nanoslits.

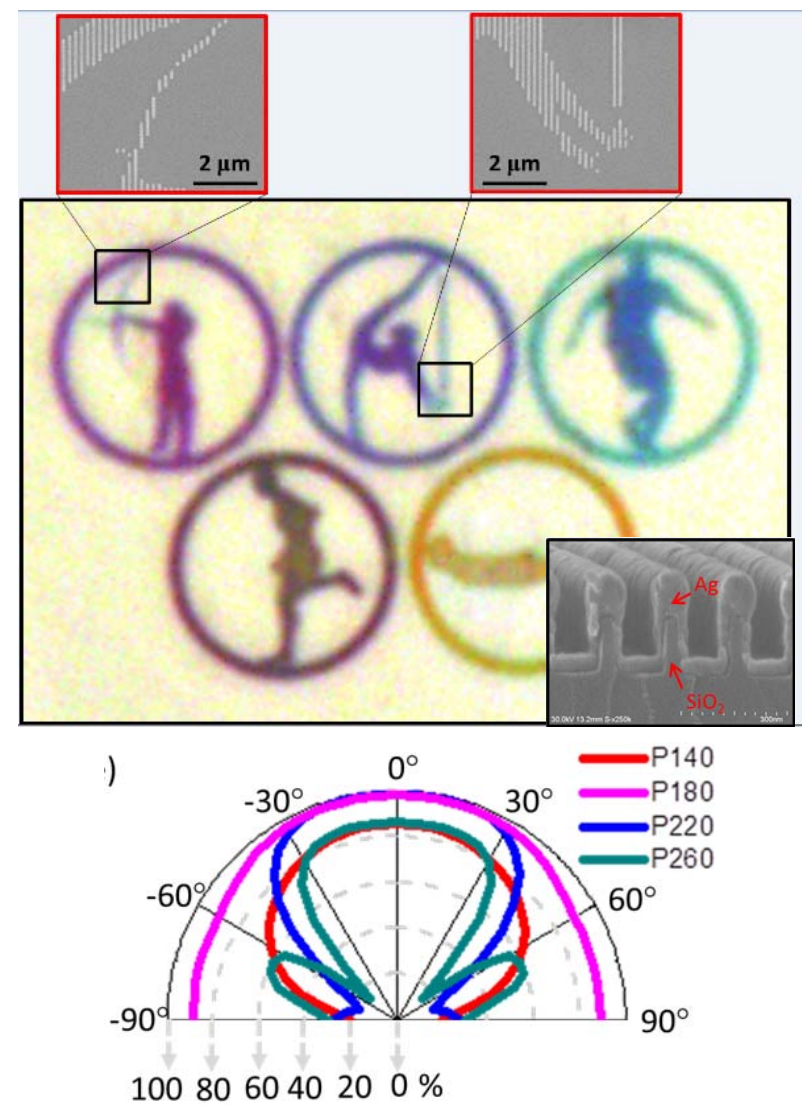

Figure 3. The SEM images and optical image under white light illumination of fabricated colored Olympic rings. The nanoresonator is formed by coating Ag layer over a dielectric nanogratings, forming metallic nanoslits. The full range of CMY colours are achieved by sweeping the width of the slit from 40 to $90 \mathrm{~nm}$, with period and depth fixed at $180 \mathrm{~nm}$ and $170 \mathrm{~nm}$ respectively. Bottom figure shows that the angleindependent color appearance can be obtained with certain period (e.g. 180nm)
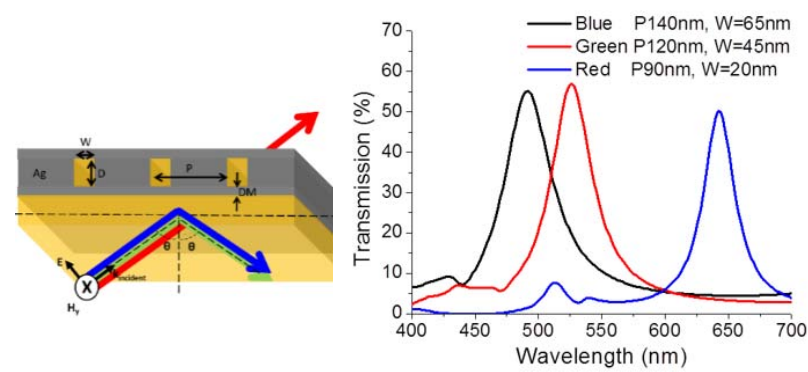

Figure 4. Schematic of transmission type filter based on metallic slit structures and parameters for RGB colors. Two layer of thin $\mathrm{Ag}$ are added in order to increase impedance contrast, which leads to improved color purity.
Similar concept can be applied to design transmission type color filters [14], e.g. by sandwiching a metal slit layer between two thin Ag layers. Based on the design in Figure 4, one can optimize the parameters of period $\mathrm{P}$, depth $\mathrm{D}$, and slit width $\mathrm{W}$ to achieve a set of blue, green, and red color filters which have good color purity and color tunability.

\section{Angle-independent reflection color filters based on resonators with lossy dielectrics}

The above structure has achieved satisfactory angleinsensitivity, but it requires a number of serial fabrication steps and it can be difficult to fabricate deep nanoscale grooves over a large area, which may limit the practical applications. Now we are exploring new designs based on multi-layer stack structures. Here we present an example of a reflective spectrum filters with angle-tolerance based on strong resonance effect in an ultra-thin absorbing material.

Strong interference effects in lossy medium have recently been demonstrated, where the thickness of cavity layer can be reduced drastically as compared to that of traditional cavity consisting of a transparent material $[15,16]$. This is due to the large absorption coefficient of the lossy material and the nontrivial phase change from the reflection at the boundary between lossy material and metal. With these properties, a resonance behavior was observed in the structure that is composed of a few nanometer thick germanium $(\mathrm{Ge})$ layer on top of the gold $\mathrm{Au}$ ) substrate. However the bandwidth of the device is broad, showing poor quality factor and hence the low color purity.

Based on a similar principle, we are able to considerably improve the color purity by choosing a highly absorbing material that has a lower complex refractive index than Ge and
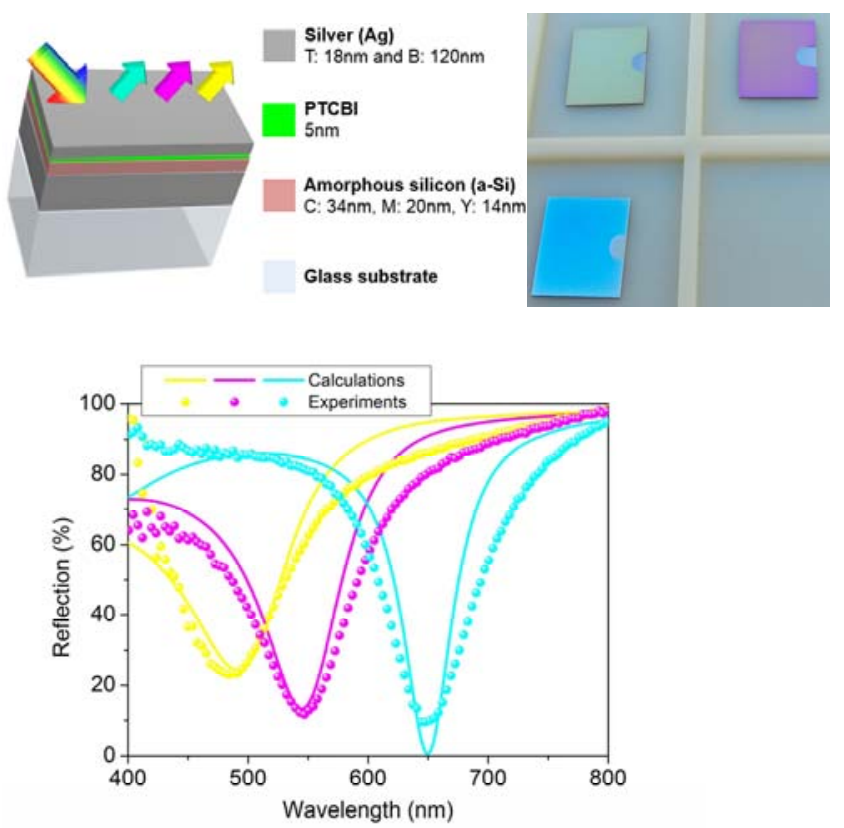

Figure 5. (a) Schematic of the angle robust spectrum filters and (b) the optical images of the fabricated samples reflecting $\mathrm{C}, \mathrm{M}, \mathrm{Y}$ colors. (c) Measured and calculated reflection spectra at oblique angles of incidence ranging from $15^{\circ}$ to $65^{\circ}$ for TM polarization. 
putting additional thin metal layer on top of the structure to increase the reflection from the top surface. The structural parameters for individual colors are designed by using numerical simulation. In addition, our devices are capable of filtering incident white light into a particular color with low sensitivity to the angle of incidence up to $\pm 65^{\circ}$, which is confirmed by both simulation and experiment [17]. The device structure comprises a highly absorbing material sandwiched by a top thin and bottom thick metal layers, all of which are built on the fused silica substrate. Amorphous silicon (a-Si) is chosen as a lossy material since the complex refractive index of a-Si is lower than that of Ge. This relatively low index can lead to the low light absorption [18], thereby increasing the Qfactor, while still maintaining the strong interference effects in ultra-thin regimes. Furthermore, the silver $(\mathrm{Ag})$ is employed for both top and bottom metal layers owing to its lowest absorption loss and highest reflection across the whole visible wavelength ranges. The thickness of top $\mathrm{Ag}$ film is designed to be $18 \mathrm{~nm}$ that allows the incident light to traverse the structure and simultaneously provides an enhanced reflection, whereas the bottom Ag is $120 \mathrm{~nm}$ to block the transmitted light. The angleinsensitive color is expected because the resonance is created in the ultra-thin absorbing layer, where the phase accumulated as the light propagates through the ultrathin film is insignificant as compared to the non-trivial reflection phase shifts. This property has been confirmed in our experiment. Similar principle can also be applied to design transmission type color filters.

\section{Discussion}

The next step for the research on the structural color devices will focus on two aspects: first, continued improvement of their optical performances, including the transmission and reflection efficiencies, the purity of filtered color, and the incident angleindependency. Second, the development of a more efficient, high-throughput nanofabrication method to realize mass production with low costs, such as roll-to-roll nanoimprint technique [19]. Newer structures should be pursued that could allow multiple colors be fabricated in parallel with only one or few different process steps. This will represent significant reduction in the fabrication complexity and will reduce the manufacturing cost. These structural colored elements could open up a bright future for the next generation high-resolution display, spectral imaging, and environmentally friendly colored coatings.

\section{Impact}

This paper discussed a number of structural color designs that can find potential applications in displays and in e-papers. Some of the structures can also be developed as pigments for coating but without involving chemicals. Furthermore, the structures that have the form of metal-dielectric-metal can be exploited for electro-optic applications, e.g. by using light-sensitive materials such as semiconductors. Indeed color filters capable of generating electric power by using harvested energy have been demonstrated [10], as well as colored solar panel that can be used as decorative signage displays [20].

\section{Acknowledgement}

The work is supported by the NSF ECCS, and was performed by Ting Xu, Alex Kaplan, Yi-Kuei Wu, Kyu-Tae Lee and Cheng Zhang, whose efforts are greatly appreciated.

\section{References}

[1] T. Xu, H.-F. Shi, Y.-K. Wu, A. F. Kaplan, J. G. Ok, and L. J. Guo, "Structural Colors: from Plasmonic to Carbon Nanostructures," Small, 7, 3128 (2011).

[2] Zi, J. et al. Coloration strategies in peacock feathers. Proc. Natl. Acad. Sci. U. S. A. 100, 12576, (2003).

[3] Vukusic, P., Sambles, J. R. \& Lawrence, C. R. Structural colour - Colour mixing in wing scales of a butterfly. Nature 404, 457. (2000)

[4] S. Kinoshita, S. Yoshioka, and J. Miyazaki, Reports on Progress in Physics 71, 076401 (2008).

[5] S. Yokogawa, S. P. Burgos, and H. A. Atwater, Nano Letters 12 (2012).

[6] E. Laux, C. Genet, T. Skauli, and T. W. Ebbesen, Nature Photonics 2 (2008).

[7] C. C. Fesenmaier, Y. Huo, and P. B. Catrysse, Opt. Express 16, 20457, (2008).

[8] A. Kaplan, T. Xu, and L. J. Guo, "High Efficiency ResonanceBased Color Filters with Tunable Transmission Bandwidth Fabricated Using Nanoimprint Lithography," Appl. Phys. Lett. 99, (2011).

[9] J. B. Guo, C. M. Huard, Y. Yang, Y. J. Shin, K. T. Lee, L. J. Guo, "Compact and ITO-Free Liquid Crystal Devices using integrated structural Color Filters and Graphene Electrode," Adv. Opt. Mater, in press, 2014. DOI: 10.1002/adom.201300525

[10] H. J. Park, T. Xu, J. Y. Lee, A. Ledbetter, and L. J. Guo, "Photonic Color Filters Integrated with Organic Solar Cells for Energy Harvesting," ACS Nano, 9, 7055, (2011).

[11] Aksu, S. et al. High-Throughput Nanofabrication of Infrared Plasmonic Nanoantenna Arrays for Vibrational Nanospectroscopy. Nano Letters 10, 2511-2518 (2010).

[12] Ross, B. M., Wu, L. Y. \& Lee, L. P. Omnidirectional 3D Nanoplasmonic Optical Antenna Array via Soft-Matter Transformation. Nano Letters 11, 2590-2595 (2011).

[13] Y.-K. Wu, A. E. Hollowell, C. Zhang, L. J. Guo, "AngleInsensitive Structural Colours based on Metallic Nanocavities and Coloured Pixels beyond the Diffraction Limit," Scientific Reports, 3, 1194, (2013).

[14] J. Zhou, and L. J. Guo, "Transition from a spectrum filter to a polarizer in a metallic nano-slit array,” Sci. Rep. 4, 3614, 2014.

[15] M. A. Kats, R. Blanchard, P. Genevet, and F. Capasso, Nature Mater. 12, 20-24 (2013).

[16] M. A. Kats, S. J. Byrnes, R. Blanchard, M. Kolle, P. Genevet, J. Aizenberg, and F. Capasso, Appl. Phys. Lett. 103, 101104 (2012).

[17] K. T. Lee, S.-Y. Seo, J.Y. Lee, and L. J. Guo, "Angle robust spectrum filters employing highly absorbing materials," in preparation, 2014.

[18] L. A. A. Pettersson, L. S. Roman, and O. Inganas, J. Appl. Phys. 86, 487-496 (1999).

[19] S. H. Ahn, and L. J. Guo, "High Speed Roll-to-Roll Nanoimprint Lithography on Flexible Plastic Substrate," $A d v$. Mater, 20, 2044, 2008; "Large-area Roll-to-Roll and Roll-toPlate Nanoimprint Lithography and analytical models for predicting residual layer thickness," ACS Nano 3, 2304, 2009.

[20] J. Y. Lee, K. T. Lee, S.Y. Seo, L. J. Guo, "Decorative power generating panels creating angle insensitive transmissive colors," Scientific Reports 4, 4192, 2014. 